OPEN ACCESS

Edited by: Paolo Palma

Bambino Gesù Children Hospital (IRCCS), Italy

Reviewed by:

Veronica Santilli,

Bambino Gesù Children Hospital (IRCCS), Italy

Alberto Cagigi, Karolinska Institutet (KI), Sweden

${ }^{*}$ Correspondence:

Agnieszka Piekarska agnieszka.piekarska@gumed.edu.pl orcid.org/0000-0002-1593-8005

Specialty section:

This article was submitted to Vaccines and Molecular Therapeutics,

a section of the journal

Frontiers in Immunology

Received: 23 July 2020 Accepted: 28 October 2020 Published: 24 November 2020

Citation:

Piekarska A, Wisniewski P, Lewandowski K, Gil L, Trzonkowski P, Bieniaszewska $M$ and Zaucha JM

(2020) Immune Status Against Hepatitis B in Patients After Allogeneic Hematopoietic Cell Transplantation-

Factors Affecting Early and Long-

Lasting Maintenance of Protective Anti-HBs Titers.

Front. Immunol. 11:586523. doi: 10.3389/fimmu.2020.586523

\section{Immune Status Against Hepatitis B in Patients After Allogeneic Hematopoietic Cell Transplantation- Factors Affecting Early and Long- Lasting Maintenance of Protective Anti-HBs Titers}

\section{Agnieszka Piekarska ${ }^{1 *}$, Piotr Wisniewski ${ }^{2}$, Krzysztof Lewandowski $^{3}$, Lidia Gil $^{4}$,} Piotr Trzonkowski ${ }^{5}$, Maria Bieniaszewska ${ }^{1}$ and Jan Maciej Zaucha ${ }^{1}$

\begin{abstract}
${ }^{1}$ Department of Hematology and Transplantology, Medical University of Gdansk, Gdansk, Poland, ${ }^{2}$ Department of Endocrinology and Internal Diseases, Medical University of Gdansk, Gdansk, Poland, ${ }^{3}$ Department of Laboratory Medicine, Medical University of Gdansk, Gdansk, Poland, ${ }^{4}$ Department of Hematology and Stem Cell Transplantation, Poznan University of Medical Sciences, Poznan, Poland, ${ }^{5}$ Department of Clinical Immunology, Medical University of Gdansk, Gdansk, Poland
\end{abstract}

The immunization of allogeneic hematopoietic cell transplantation (HCT) recipients against vaccine-preventable diseases is a part of posttransplantation guidelines. We conducted a prospective study to assess clinical and immunological parameters that would determine the response and long-term maintenance of protective antibody titers upon the hepatitis $B$ virus (HBV) vaccination after HCT. The investigated variables included: vaccination of the HCT recipients and their donors prior to HCT, chronic graft versus host disease (cGVHD) and the timing of post-HCT vaccination, and B- and T-cell subtype status. Forty-two patients were immunized with three or more doses of recombinant hepatitis $B$ surface antigen (rHBsAg) administered according to the individualized schedule of 0-1-2-6-(12) months. After vaccination, seroconversion was achieved in the whole group. The vaccines were categorized according to the antibody (Ab) titers as weak (WRs; 28.7\%), good (GRs; $38 \%$ ) or very good responders (VGRs; 3.3\%). In multivariate logistic regression, severe cGVHD (OR= 15.5), and preceding donor immunization $(\mathrm{OR}=0.13)$ were independent predictors of a weak response to vaccination. A prior belonging to the WR group impaired the durability of protection $(\mathrm{OR}=0.17)$ at a median follow-up of 11.5 years. Patients with severe CGVHD showed a trend toward lower median Ab titers, although they required a higher rate of booster vaccine doses. All VGRs had CD4+ cells $>0.2 \times 10^{6} / \mathrm{L}$. There was a lower mean rate of CD4+IL2+ lymphocytes in WRs. Vaccination demonstrated the immunomodulatory effect on B-cell and T-cell subsets and a Th1/Th2 cytokine profile, while shifts depended on a history of severe cGVHD and the type of vaccine responder. To conclude, vaccination of HCT donors against HBV allows a better response to vaccination in the respective HCT recipients. Double doses of $\mathrm{rHBsAg}$ should be considered in 
patients with CGVHD and in those not immunized before HCT. A dedicated intensified vaccination schedule should be administered to WRs.

Keywords: hepatitis B vaccine, vaccination schedule, donor vaccination, hematopoietic cell transplantation (HCT), chronic graft versus host disease (GVHD)

\section{INTRODUCTION}

Allogeneic hematopoietic cell transplantation (HCT) is a curative cellular therapy for a variety of disorders (1). In posttransplantation care, a dysfunctional immune system and infectious complications pose serious problems (2-5). Moreover, an observed gradual loss of specific postvaccination immunity after HCT necessitates the immunization of HCT recipients against vaccine-preventable diseases $(6,7)$. Following HCT, hepatitis $\mathrm{B}$ virus $(\mathrm{HBV})$ can trigger serious liver complications, including fulminant hepatitis. Repetitive exposure to medical procedures poses a risk of $\mathrm{HBV}$ transmission. Moreover, reverse seroconversion upon immunosuppressive treatment is reported in anti-HBc-positive patients in whom $\mathrm{HBV}$ infection was resolved before HCT $(8,9)$. Therefore, in countries with a high incidence of hepatitis $\mathrm{B}$, immunization with recombinant hepatitis B surface antigen ( $\mathrm{rHBsAg}$ ) and the maintenance of protective anti-HBs antibodies (Abs) are especially justified $(6,10)$.

Due to deficiencies in humoral and cellular immunity, as well as altered mechanisms regulating immune reactions, the overall response rate (ORR) of transplant recipients is inferior compared to that of healthy people (11-15). However, for the sake of simplicity, a universal vaccination protocol for all transplant recipients does not consider differences in immune recovery in the distinct HCT platforms (16-21). Data on the durability of postvaccine protection in HCT recipients are limited. Longlasting immunity depends on many variables, including the immunogenicity of vaccines, immunosuppressive treatment, and chronic graft versus host disease (cGVHD) $(18,19)$.

The clinical presentation of cGVHD mimics autoimmune diseases, and the organ-debilitating impact does not spare the immune system (22-26). Weak granulocyte chemotaxis, low response to mitogens, defects of the primary and secondary immune response to bacterial and polysaccharide antigens as well as functional hyposplenism are well-known phenomena $(27,28)$. A state of chronic inflammation, maintained by IL17, may lead to immune exhaustion, while dysregulated polyclonally activated lymphocytes do not properly recognize specific antigens $(23,29)$. Therefore, severe cGVHD itself could deteriorate the postvaccine immune responses and the maintenance of anti-HBV immunity.

We launched a prospective study aiming at identification of clinical and immunological factors that determine the response and long-term maintenance of protective antibody titers upon individualized vaccination with $\mathrm{rHBsAg}$ after HCT considering: donor/recipient serological anti-HBV status, incidence and severity of cGVHD, the timing of vaccination after HCT and the patient immune reconstitution. The serological monitoring included antiHBs Ab levels tested before HCT, and after transplantation up to rHBsAg administration and postvaccination follow-up.
Subpopulations of B-cell and T-cell compartments, as well as the cytokine Th1/Th2 profile, were evaluated in the perivaccination period. An additional goal of the study was to optimize a vaccination schedule and standardize posttransplantation antiHBV surveillance.

\section{MATERIAL AND METHODS}

\section{Patients}

Criteria to initiate a vaccination program included a lack of previous vaccination after allogenic HCT, the remission of any underlying disease, the discontinuation of immunosuppressive treatment for at least 2 months before vaccination, a lack of active infection and signed informed consent. Patients with a history of cGVHD were accepted, provided that they did not suffer from an active disease requiring immunosuppressive therapy. Altogether, 62 Caucasian patients qualified, but for the homogeneity of the study group, patients allotransplanted with the use of reduced intensity/toxicity conditioning regimens were excluded from the final analysis.

Standard GVHD prophylaxis consisted of cyclosporine and a short course of methotrexate. Anti-thymocyte globulin administration $(7.5 \mathrm{mg} / \mathrm{kg})$ was a component of GVHD prophylaxis in HCT from matched unrelated donors (MUDs).

A final study group consisted of 48 patients who started a vaccination program between Dec 2003 and Mar 2006, including 42 individuals requiring vaccination with rHBsAg. The study was designed to compare the quality of the immune response between patients immunized in the early (between 6 and 24 months) and late periods (> 24 months) after transplantation.

\section{Vaccination Protocol}

Vaccination against HBV was a part of the whole immunization schedule, consisting of vaccines against poliomyelitis (3 doses), tetanus (3 doses), diphtheria (3 doses), Haemophilus influenzae (2 doses), and HBV, administered simultaneously in separate parts of the body. Immunization against influenza was given seasonally once a year, while immunization against Streptococcus pneumoniae was performed with a 23 -valent polysaccharide vaccine $\geq 12$ months post-HCT, as conjugated vaccines were not available at that time (11).

The recombinant surface antigen of $\mathrm{HBV}$ gained from Saccharomyces cerevisiae and absorbed on aluminum compounds was used (Engerix B; GlaxoSmithKline Biologicals). The protocol consisted of 3 or more doses of the vaccine administered intramuscularly in 4- to 6-week intervals according to the following schedule: 0-1-2-6-(12) months. The first dose of the vaccine in every case was double $(40 \mu \mathrm{g})$ the standard dose. The titer of anti-HBs Abs was checked 4 - 6 weeks after every dose, and the administration of 
subsequent doses depended on the grade of the response. A lack of seroconversion or a low titer of $\mathrm{Abs}$ (anti-HBs $<10 \mathrm{mIU} / \mathrm{ml}$ ) was followed by subsequent administration of a double vaccine dose until a titer of anti-HBs Abs $>10 \mathrm{mIU} / \mathrm{ml}$ was achieved. In the case of seroconversion or an anti-HBs Ab titer $>10 \mathrm{mIU} / \mathrm{ml}$ after the initial dose, the next doses were single doses $(20 \mu \mathrm{g})$. After protocol completion, the anti-HBs Ab titer was monitored regularly during visits in the posttransplantation unit. Revaccination was prescribed in patients in whom protective immunity was lost, including patients with anti-HBc positivity.

Patients were divided into three types of responders, weak (WRs), good (GRs), and very good responders (VGRs), depending on the achieved titer of anti-HBs Abs, the administered vaccine doses, and the maintenance of protective levels of humoral anti-HBV immunity (Table 1).

\section{Chronic GVHD}

Chronic GVHD diagnosis was based on data from patient medical records, and the criteria of NIH 2014 Consensus were retrospectively adopted (26).

\section{Flow Cytometry}

Immunophenotyping was performed from heparinized peripheral blood according to standard procedures at least 3 times: before vaccination and during and after the completion of the basic vaccination protocol. Analyses were performed using triple-color flow cytometry (FC). All Abs used for immunofluorescent staining were obtained from Becton Dickinson (BD), and cells were acquired on a FACS Calibur (BD).

Cells were incubated with the following Ab-conjugates: $\gamma 1 / \gamma_{2} \mathrm{a}-$ FITC/PE (clone X39/X40), CD3-PerCP (clone SK7), CD4-PE (clone SK3), CD4-PerCP (clone SK3), CD8-FITC (clone SK1), CD8PerCP (clone SK1), CD45RA-FITC (clone L48), CD45RO-PE (clone UCHL-1), CD19-CyChrome (clone HIB19), CD27-PE (clone L-128), IgD-FITC (clone IA6-2), IgM-FITC (clone G20127), IgG-FITC (clone G18-145), IFN $\gamma$-FITC (clone 25723.11), IFN $\gamma$-PE (clone 25723.11), IL2-FITC (clone 5344.111), IL2-PE (clone 5344.111), IL4-PE (clone 3010.211), IL5-PE (clone JES1.39D10), and IL10-PE (clone JES3.12G8).

Assessments of T-cell subsets were performed in whole blood, while for assessments of B-cell subsets and cytokine expression, peripheral blood mononuclear cells (PBMCs) were isolated. Cellular subpopulations were analyzed in the lymphocyte gate by positive signals above the isotype fluorescent control.

TABLE 1 | Criteria for the WR, GR, or VGR groups depending on the achieved anti-HBs titers, the quantity of injected doses, and the maintenance of high protective immunity.

\begin{tabular}{ll}
\hline Groups & \multicolumn{1}{c}{ Criteria } \\
\hline Weak responders & $-\geq 4$ doses to achieve anti-HBs Abs 10-100 mlU/ml or \\
(WRs) & - anti-HBs Abs $>100 \mathrm{mlU} / \mathrm{ml}$ achieved after 2-4 doses but \\
& maintained no longer than 1 year \\
Good responders & - anti-HBs Abs $>100 \mathrm{mlU} / \mathrm{ml}$ achieved after 3-4 doses and \\
(GRs) & maintained at least 1 year \\
Very good & - anti-HBs Abs $>100 \mathrm{mlU} / \mathrm{ml}$ achieved after 1-2 doses and \\
responders (VGRs) & maintained at least 1 year
\end{tabular}

\section{Isolation of PBMCs}

PBMCs were separated by density gradient centrifugation on a Histopaq 1077 (Sigma). After washing in RPMI medium (Tominex) mixed with 10\% FBS medium (Tominex), cellularity was assessed, and PBMCs were suspended in RPMI/FBS medium to achieve a lymphocyte concentration of $2 \times 10^{6} / \mathrm{ml}$. Some isolated lymphocytes were suspended in the medium for cell culture, and the rest were stained with Abs against B-cell markers.

\section{Stimulation of T-lymphocytes with PMA and Ionomycin}

Isolated lymphocytes were incubated with phorbol 12-myristate 13-acetate (PMA; Sigma-Aldrich) and ionomycin (SigmaAldrich) in the presence of an inhibitor of cytokine secretion, Brefeldin A (GolgiPlug; BD), in round-bottom 24-well plates for $5-6 \mathrm{~h}$ at $37^{\circ} \mathrm{C}$ in a $5 \% \mathrm{CO}_{2}$ atmosphere according to a standard protocol from Laboratoire d'Immunologie CHU Rangueil (Toulouse, France), with modifications implemented by the first author $(30,31)$.

\section{Staining of Surface Antigens and Intracellular Cytokines}

The staining of membrane antigens from whole blood was preceded by double lysis. Cell suspensions were incubated with cocktails of antibodies/conjugates for $30 \mathrm{~min}$ in darkness and then washed in PBS (5 min; RCF 650). After centrifugation, the supernatant was removed, and specimens were ready for acquisition. Samples requiring intracellular staining were fixed with 3\% formaldehyde and permeabilized with Perm2 (BD). After 30 min of incubation in darkness with cytokine-targeting Abs/conjugates, cells were washed and suspended in $200 \mu \mathrm{l}$ of $0.5 \%$ formaldehyde solution. Acquisitions with FC were performed within $24 \mathrm{~h}$.

\section{Ethical Approval}

This study was performed in accordance with the Declaration of Helsinki and received the approval of the Independent Bioethics Committee of the Medical University of Gdansk. Informed consent forms were signed by all participating patients, and the possible consequences of the study were fully explained.

\section{Statistical Analysis}

Data analysis involved descriptive statistics, contingency tables, Pearson's chi-square test and the Mann-Whitney-Wilcoxon U test. Logistic regression was used to examine the influence of selected predictors jointly. A p-value of less than 0.05 was considered significant. All statistical analyses, data manipulation, and graphical plots were performed using the RStudio statistical software environment (version 1.1 with R.3.6.1).

\section{RESULTS}

\section{Patients' Characteristics}

The median ages at transplantation and vaccination were 35 and 39 years, respectively. Forty-two patients immunized with rHBsAg were divided into the early (38\%) and late vaccination groups (62\%) 
according to the time elapsed after HCT. Table 2 presents detailed patient characteristics. Five patients with naturally acquired antiHBV immunity and one patient with solely adoptive anti-HBV immunity transfer were included in serological monitoring (the socalled 'initially nonvaccinated group').

The evaluation of long-lasting anti-HBV immunity was possible in 43 patients. Five patients were excluded due to a short follow-up. The median follow-up in the analyzed vaccinated group was 11.5 (range, 5-16) years, while in the initially nonvaccinated group, it was 16.5 (range, 15-21) years.

\section{Immune Status Against HBV \\ Serological Status of Patients and Donors}

Thirty $(62.5 \%)$ patients were vaccinated with rHBsAg before HCT, with an ORR of 53\%, including 6 patients achieving an anti-HBs Ab range of $10-100 \mathrm{mIU} / \mathrm{ml}$ and 10 patients with anti$\mathrm{HBs} \mathrm{Ab}$ levels $>100 \mathrm{mIU} / \mathrm{ml}$. Five patients with naturally acquired immunity were anti- $\mathrm{HBc}$ and anti-HBs $\mathrm{Ab}$ positive (>100 mIU/ml). In $20(41.7 \%)$ patients with anti-HBs Abs $<10$ $\mathrm{mIU} / \mathrm{ml}$, passive immunization with anti-HBs gamma-globulin was administered.

Twenty-five (58\%) of 43 matched sibling donors (MSDs) were vaccinated before donation with an ORR of $60 \%$, and 3 donors had protective anti-HBs $\mathrm{Ab}$ titers following $\mathrm{HBV}$ infection. In 19 (44.2\%) HCTs from MSDs, both donors and recipients were vaccinated.

\section{Maintenance of Anti-HBV Protection Post-HCT Before Vaccination}

Three months, 6 months, and 1 year after HCT, anti-HBs Abs were detected in $87 \%, 69 \%$, and $40 \%$ of patients, respectively

TABLE 2 | Patient characteristics.

\begin{tabular}{lc}
\hline Age (at transplantation); median (range) years & $35(16-54)$ \\
Age (at vaccination); median (range) years & $39(19-57)$ \\
Sex: female/male & $22 / 26$ \\
Primary disease: & \\
$\quad$ AML/ALL & 18 \\
CML & 26 \\
$\quad$ Other (MDS, PNH, CEL) & 4 \\
Chemotherapy preceding HCT: Yes/No & $18 / 30$ \\
Conditioning regimen: TBICy/BuCy120 & $9 / 39$ \\
Type of donor: MUD/MSD & $6 / 42$ \\
Source of hematopoietic cells: BM/PB & $14 / 34$ \\
CD34+ cells dose (x10 $\%$ /kg recipient body weight): & \\
BM median (range) & $3.3(1.2-5.4)$ \\
PB median (range) & $6.5(2.7-8.9)$ \\
aGvHD grade 2-3 (\%) & $21(43.8 \%)$ \\
cGVHD & $30(62.5 \%)$ \\
cGVHD no/mild/moderate/severe & $18 / 9 / 10 / 11$ \\
CMV reactivations/median months post-HCT (range) & $17(35.4 \%) / 4(1-12)$ \\
Immunization with rHBsAg: total; early vs. late group & $42 ; 16(38 \%)$ vs. 26 (62\%)
\end{tabular}

AML, Acute Myeloid Leukemia; ALL, Acute Lymphoblastic Leukemia; CML, Chronic Myeloid Leukemia; MDS, Myelodysplastic Syndrome; PNH, Paroxysmal Nocturnal Hemoglobinuria; CEL, Chronic Eosinophilic Leukemia; MUD, matched unrelated donor; MSD, matched sibling donor; BM, bone marrow; PB, peripheral blood; HCT, Hematopoietic Cell Transplantation; aGVHD, acute graft versus host disease; cGVHD, chronic graft versus host disease; CMV, cytomegalovirus; rHBsAg, recombinant hepatitis $B$ surface antigen.
(Table 3). Protective anti-HBs Ab levels > $10 \mathrm{mIU} / \mathrm{ml}$ were found 3 months, 6 months and 1 year after HCT in 42\%, 33\%, and $15 \%$ of patients, respectively.

Three months post-HCT, there were no significant differences in anti-HBV protection between those immunized actively and those immunized passively.

Six months post-HCT, in univariate analysis, anti-HBs Abs were detectable significantly more often in those vaccinated before HCT $(p=0.025)$ or in the case of donor vaccination $(\mathrm{p}=0.006)$. In multivariate analysis, the odds of having anti$\mathrm{HBV}$ protection (anti-HBs Abs levels $>10 \mathrm{mIU} / \mathrm{ml}$ ) depended on a recipient vaccination prior to HCT (OR 8.9, 95\% CI: $1.4-$ $177.3 ; \mathrm{p}=0.052$ ), or naturally acquired immunity (OR 13.1, 95\% CI: $1.3-321.8 ; \mathrm{p}=0.048$ ). Other analyzed predictors (passive immunization, donor immunization) were not significant in multivariate analysis.

In univariate analysis, the maintenance of anti-HBV immunity at one year was significantly dependent on anti-HBs $\mathrm{Ab}$ levels $>10 \mathrm{mIU} / \mathrm{ml}$ in donors $(\mathrm{p}=0.006)$ and prior effective vaccination in the patients $(\mathrm{p}=0.04)$. In multivariate analysis, naturally acquired anti-HBV immunity (anti-HBc positivity, anti-HBs positivity) increased the odds of maintaining protective anti-HBs titers (OR 5.09, 95\% CI: 0.9-32.0; $\mathrm{p}=$ 0.065 ). The remaining predictors (passive immunization, donor's immunization) were not significant in multivariate analysis. The graphical presentation of anti-HBV protection up to one-year post-HCT with respect to recipient and donor antiHBV immunity status is presented in Figure 1.

No reverse seroconversion was observed in the anti- $\mathrm{HBc}$ and anti-HBs Ab-positive patients. The adoptive transfer of antiHBV immunity occurred in 10 patients with grafts from MSDs.

\section{Response to Vaccination With rHBsAg: Patient-, Transplant-, and Donor-Related Factors}

Seroconversion was achieved in the whole group. There were 12 (28.7\%) patients classified as WRs, $16(38 \%)$ as GRs, and 14 (33.3\%) as VGRs. No severe complications related to the immunization of HCT recipients were recorded.

Statistical analysis did not show the influence of various factors, including treatment with chemotherapy preceding HCT, the type of conditioning regimen, the source of hematopoietic cells, the type of donor, the patient's previous vaccinations, reactivation of cytomegalovirus, of the patient's age at transplantation and vaccination, on the results of active immunization after HCT in the study group. In univariate analysis, there was a trend toward the unfavorable impact of

TABLE 3 | Maintenance of anti-HBV protection in the prevaccination and prerevaccination period post-HCT.

\begin{tabular}{lccc}
\hline $\begin{array}{l}\text { Timepost- } \\
\text { HCT }\end{array}$ & $\begin{array}{c}\text { Detectable anti-HBs } \\
\text { antibodies* }\end{array}$ & $\begin{array}{c}\text { Anti-HBs 10-100 } \\
\text { mIU/ml }\end{array}$ & $\begin{array}{c}\text { Anti-HBs > } \\
\mathbf{1 0 0} \mathbf{m I U} / \mathbf{m l}\end{array}$ \\
\hline 3 months & $87 \%$ & $42 \%$ & $29 \%$ \\
6 months & $69 \%$ & $33 \%$ & $12 \%$ \\
1 year & $40 \%$ & $15 \%$ & $8 \%$ \\
\hline
\end{tabular}

*Including all patients with anti-HBs titer $\geq 0.5 \mathrm{~m} / \mathrm{U} / \mathrm{ml}$. 


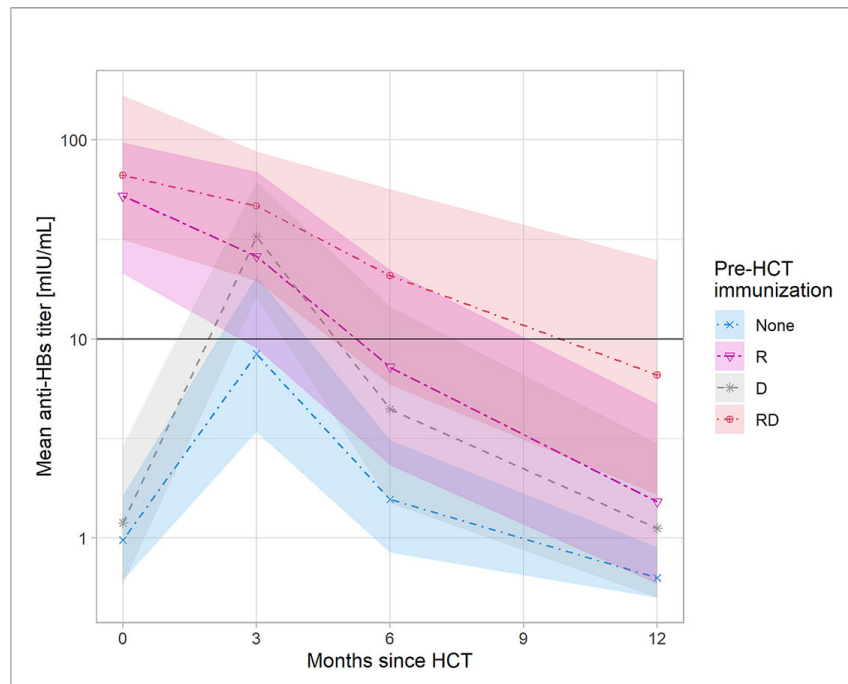

FIGURE 1 | Anti-HBV protection up to one year post-HCT with respect to recipient and donor anti-HBV immunity. The shaded areas represent the 95\% confidence intervals. Linear charts represent mean values of anti-HBs titers with respect to the anti-HBV immunity status of recipient and donor pre-HCT: - None - recipient nonimmunized actively or immunized ineffectively (anti$\mathrm{HBs}<10 \mathrm{mlU} / \mathrm{ml}$ ) and donor nonimmunized, - R - only recipient immunized, $D$ - only donor immunized, - RD - both recipient and donor immunized, A rise of anti-HBs titer 3 month post-HCT is caused by passive immunization with anti-HBs gamma-globulins prior to HCT administered to patients with anti$\mathrm{HBs}<10 \mathrm{mlU} / \mathrm{ml}$. severe cGVHD on the results of active immunization with rHBsAg $(\mathrm{p}=0.057)$. Seroconversion after the first vaccine dose was significantly more frequent in patients who received transplants from donors immunized against HBV $(\mathrm{p}=0.022)$. The majority (91\%) of VGRs had immunized donors, in contrast to $33 \%$ of WRs ( $\mathrm{p}=0.018$ ). In 11 patients, adoptive immunity transfer was noted. Kaplan-Meier analysis indicated the positive impact of adoptive immunity transfer on postvaccination responses $(p=0.014)$. In the multivariate model, severe cGVHD increased the odds for WRs (OR=15.5, 95\% CI: 1.9244.0; $\mathrm{p}=0.02$ ), while preceding donor immunization decreased the odds for WRs (OR= 0.13, 95\% CI: 0.01-0.9; $\mathrm{p}=0.05)$. A time of immunization $\geq 24$ months after HCT (the late vaccination group) was inversely associated with weak response (OR $=0.43$, 95\% CI: 0.04-3.5; $\mathrm{p}=0.4$ ), but the effect did not reach statistical significance. Other analyzed predictors (sex, age $>40$, patient immunization before HCT) did not show an influence in multivariate analysis. The chart visualizing the study group and significant differences between the response groups is presented in Figure 2.

\section{Evaluation of Long-Term Immunity}

In the long-term study group anti-HBs $\mathrm{Ab}$ concentrations $>100$, $50-99$, and $10-49 \mathrm{mIU} / \mathrm{ml}$ were detected in $30(69.8 \%), 8$ (18.6\%), and $5(11.6 \%)$ patients, respectively.

In the vaccinated group, the median anti-HBs $\mathrm{Ab}$ titer was $230 \mathrm{mIU} / \mathrm{ml}$ (range, $11->1,000 \mathrm{mIU} / \mathrm{ml}$ ). Thirteen (35.1\%)

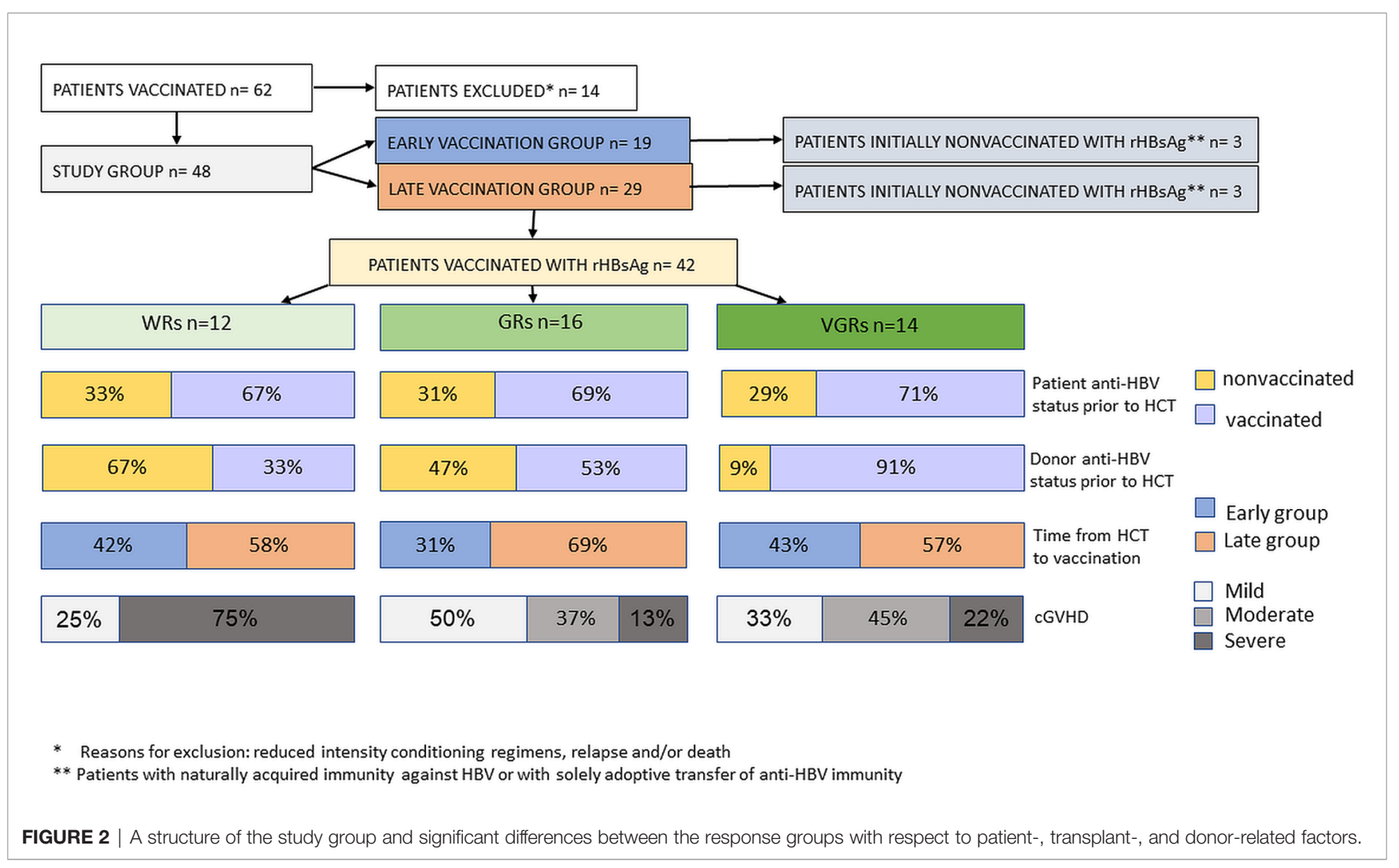


patients required reimmunization with booster doses of $\mathrm{rHBsAg}$ due to a decline in Abs. In 9 patients, one booster dose was sufficient, and 2 and 3 doses were administered in 3 and 1 patients, respectively. The majority $(84.6 \%)$ of patients achieved anti-HBs Ab titers $>100 \mathrm{mIU} / \mathrm{ml}$ upon reimmunization. There was no case of hepatitis B in the study group in the followup period.

In the initially nonvaccinated group, the median anti-HBs Ab titer was $561.5 \mathrm{mIU} / \mathrm{ml}$ (range, 91 - >1,000 $\mathrm{mIU} / \mathrm{ml}$ ). Two patients required one booster dose of $\mathrm{rHBs}$ g due to a gradual loss of immunity to approximately $30 \mathrm{mIU} / \mathrm{ml}$, and they achieved a long-term anti-HBs Ab titer > $100 \mathrm{mIU} / \mathrm{ml}$ upon reimmunization.

In univariate analysis, a prior belonging to the WR, GR, or VGR groups had a significant impact on median anti-HBs titers that were 73, 270, and $302 \mathrm{mIU} / \mathrm{ml}$, respectively $(\mathrm{p}=0.05)$. Booster rHBsAg doses were required in $9 \mathrm{WR}$ patients and in 4 GRs but in no VGR patients $(\mathrm{p}<0.0002)$. In multivariate analysis, the odds for maintaining anti-HBs titers $>100 \mathrm{mIU} / \mathrm{ml}$ were lower for the WR group $(\mathrm{OR}=0.17,95 \% \mathrm{CI}: 0.02-1.02 ; \mathrm{p}=$ 0.059). The chart visualizing the differences in the long-term immunity between the response groups and a need for booster vaccine doses is presented in Figure 3.

We observed higher median anti-HBs Ab concentrations in patients with previously noted adoptive immunity transfer (363.5 vs. $240 \mathrm{mIU} / \mathrm{ml}$ ), and fewer patients required a booster dose of rHBsAg (20\% vs. $56.6 \%)$, but statistical significance was not achieved.

The median long-term anti-HBs Ab concentrations did not differ significantly with the use of our vaccination protocol between patients vaccinated at age of $<40$ and those vaccinated at age of $\geq 40$ years $(224.5$ vs. $263 \mathrm{mIU} / \mathrm{ml}$, respectively) in univariate and multivariate analyses.

We observed higher median anti-HBs Ab titers in patients without a history of cGVHD or with mild cGVHD (282.5 and $270 \mathrm{mIU} / \mathrm{ml}$, respectively) than in patients with severe cGVHD (110 mIU/ml). In multivariate analysis, the odds for maintaining anti-HBs titers $>100 \mathrm{mIU} / \mathrm{ml}$ in patients with severe cGVHD were lower than those in patients with a mild form of or without cGVHD $(\mathrm{OR}=0.4,95 \% \mathrm{CI}: 0.04-3.07 ; \mathrm{p}=0.41)$. Although the difference was not statistically significant, in $60 \%$ of the severe cGVHD patients, the Ab titers were raised by booster doses of rHBsAg in contrast to $32 \%$ of those with a mild form of or without $c G V H D(p=0.09)$. The remaining analyzed predictors (sex, donor immunization, vaccination timing) did not show an influence in multivariate analysis.

\section{Immune Reconstitution}

The adoptive T-cell and B-cell immunity recover within months or years in patients after HCT, and this process is very individual. Immune reconstitution depends on the pretransplant factors like an underlying disease and its treatment, age, conditioning regimen, donor, a source of hematopoietic cells, and posttransplant factors, e.g., GVHD. The recovery of B-cells is similar to ontogeny and usually quantitatively normalizes around 1 year post-HCT. However, a lowered cumulation of hypermutations in VH genes, impaired isotype switch and IgG production, processes dependent inter alia on Th2 cells, are frequently observed after HCT. In contrast, T-cell reconstitution is inverted, and memory/effector T-cells dominate even many years posttransplant, while the reconstitution of naïve $\mathrm{T}$-cells, which broaden the repertoire of specificities, starts not earlier than 6 months post-HCT in the case of CD4+ cells (32).

The lowered ability to produce specific antibodies in response to vaccination, observed in a substantial proportion of HCT recipients, led to the creation of intensified vaccination schedules post-HCT, including vaccination against HBV. Upon injection, rHBsAg is lysed and processed by antigen-presenting specific Bcells and presented with MHC-II molecule to Th2 cells. Activated Th2 lymphocytes induce differentiation of B-cells to plasma cells, secreting HBsAg in high quantities to stimulate immune B-cell and T-cell memory (33). All types of immune cells involved in creating anti-HBV postvaccination immunity were included into analyses, but their reconstitution depends on the time elapsed from HCT to a great extent. Therefore, the parameters of immune reconstitution were analyzed with respect

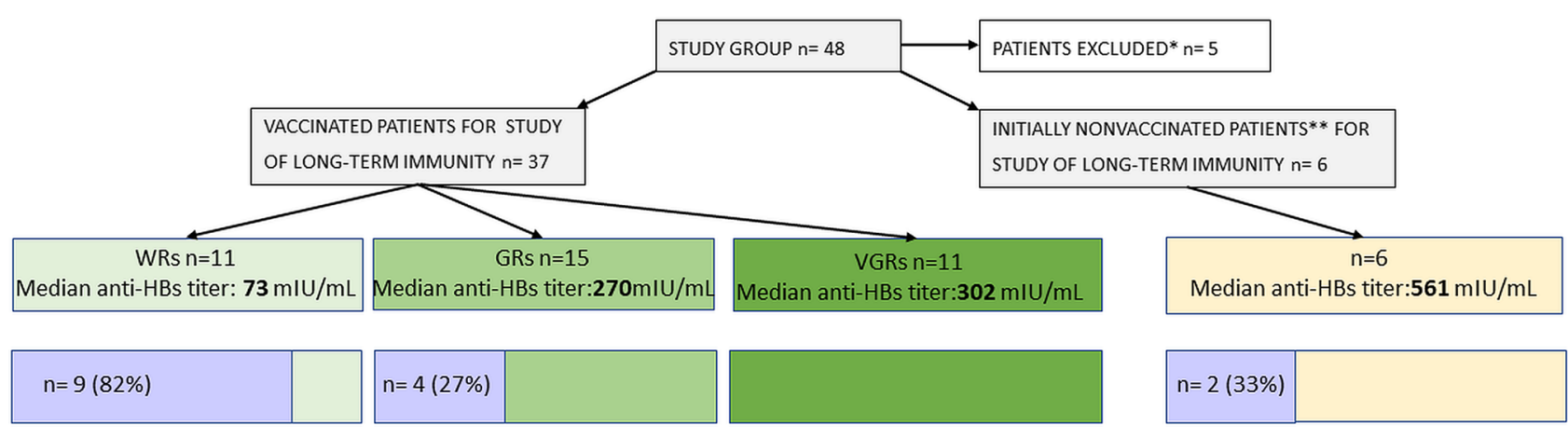

* Reasons for exclusion: lost from follow-up or too short follow-up

Booster vaccine required 
to timing from HCT and, subsequently, their impact was assessed with respect to response to vaccination with $\mathrm{rHBsAg}$.

\section{Comparison of the Early and Late Vaccination Groups}

There were no significant differences concerning absolute lymphocyte count, gamma-globulin rate or IgG and IgM levels, while the median IgA concentration was significantly higher in patients $>2$ years post-HCT ( 2.1 vs. $1.36 \mathrm{~g} / \mathrm{L} ; \mathrm{p}=0.005)$. The summarized comparison of detailed immune parameters described below is presented in Table 4 .

The reconstitution of $\mathrm{B}$ lymphocytes, including absolute counts of CD19+ cells and B-cell subpopulations with immunoglobulin $\operatorname{IgD}(+)$ and $\operatorname{IgM}(+)$ receptors, were comparable between the early and late group. The frequencies of CD19+IgG $(+)$ cells were very low in both groups, which is why only percentages are provided. Naïve (CD27-) cells dominated over memory (CD27+) B-cells ( $\mathrm{p}<$ 0.001 ), with a trend toward higher absolute values in the late group. The proportion and absolute counts of $\operatorname{IgM}(+) \mathrm{CD} 27(-)$ and $\operatorname{IgD}(+) \mathrm{CD} 27(-)$ naïve B-cells did not differ significantly between the two distinct groups, as was the case for $\operatorname{IgM}(+) \mathrm{CD} 27(+)$ and $\operatorname{IgD}(+) \mathrm{CD} 27(+)$ memory B-cells and double negative $(\mathrm{DN}) \operatorname{IgD}(-)$ CD27(-) late differentiated memory B-cells.

The reconstitution of $\mathrm{T}$ lymphocytes did not vary concerning the proportion and absolute counts of $\mathrm{CD} 3(+)$ and $\mathrm{CD} 3(+) \mathrm{CD} 8$ $(+)$ cells, while there were higher rates and absolute counts of CD3 $(+) \mathrm{CD} 4(+)$ cells in the late group: $18.2 \%\left(0.33 \times 10^{9} / \mathrm{L}\right)$ vs. $25.6 \%$ $\left(0.53 \times 10^{9} / \mathrm{L}\right)(\mathrm{p}=0.006)$. The inverse CD4/CD8 ratio was more evident in the early group: 0.52 vs. $0.86(\mathrm{p}=0.008)$. The naïve (CD45RA+CD45RO-) and memory (CD45RA-CD45RO+) subsets of $\mathrm{CD} 8(+)$ cells were comparable, while naïve $\mathrm{CD} 4(+)$ cells were less frequent than memory CD4 $(+)$ cells $(\mathrm{p}<0.001)$. In the late group, there was a trend toward more numerous naïve CD4 $(+)$ cells $(p=0.096)$, while memory CD4 $(+)$ cells achieved significantly higher values $(\mathrm{p}=0.011)$.

The expression of cytokines from the Th1 profile (IFN $\gamma+$ and $\mathrm{IL} 2+)$ dominated in both groups. IL2 predominated in CD3(+) CD8(-) cells while IFN $\gamma$ predominated in $\mathrm{CD} 3(+) \mathrm{CD} 8(+)$ cells with a trend to higher IFN $\gamma$ expression in the early group $(\mathrm{p}=0.052)$. IL2(-)IFN $\gamma(+)$ lymphocytes predominated in the early group $(\mathrm{p}=0.018)$ and $\operatorname{IL} 2(+) \operatorname{IFN} \gamma(-)$ lymphocytes were prevalent in the late group $(\mathrm{p}=0.011)$. Within the Th2 cytokine profile, the expression of IL4 was higher in CD8(-) cells, and did not differ between the early and late groups. The Th1/Th2 ratio (IL2/IL4) was slightly higher in the early group: 11.94 vs. 9.62.

\section{Variations in Parameters of Immune Reconstitution and Cytokine Profile Upon the Receipt of Vaccination}

The whole VGR group achieved an absolute count of $\mathrm{CD} 3(+)$ $\mathrm{CD} 4(+)$ cells $>0.2 \times 10^{9} / \mathrm{L}$ at vaccination. Among 6 patients who had not reached this value, half turned out to be GRs, and the remaining 3 were WRs. Insight into the naïve CD4(+) T-cell subset did not show significant differences in the immune response to vaccination for the cut-off of $0.05 \times 10^{9} / \mathrm{L}$, while there was a trend toward a lower RR for the cut-off of $0.03 \mathrm{x}$ $10 \% / \mathrm{L}$, which was not reached in $63 \%$ of WRs $(\mathrm{p}=0.057)$.

Generally, the levels of either naïve or memory T-cell compartments rose gradually during the realization of the vaccination protocol $(\mathrm{p}<0.001)$. However, in the WR group, naïve $\mathrm{CD} 4(+)$ cell counts began to rise with a delay compared to those in the GR and VGR groups $(\mathrm{p}<0.001)$. We did not find a negative impact of a history of $\mathrm{cGVHD}$ on the number of naïve CD4(+) T-cell subsets.

In turn, the numbers of CD19(+) B-lymphocyte and naïve CD27(-) and memory CD27(+) subsets had no statistical impact on the immune response to rHBsAg. Nevertheless, the rates of memory $\operatorname{IgM}(+)$ and $\operatorname{IgD}(+) C D 27(+)$ B-cell subsets showed a significant rise in the second measurement in GRs when the humoral response was observed $(\mathrm{p}=0.027$ and $\mathrm{p}=0.047$, respectively). Interestingly, the median percentages of $\mathrm{DN}$ IgDCD27- in WRs, GRs, and VGRs were 5.77\%, 4.14\%, and 5.44\% within CD19+ B cells, respectively, and the difference was significant between WRs and GRs $(p=0.047)$. Concerning a history of cGVHD, the memory B-cell subsets $\operatorname{IgM}(+)$ and $\operatorname{IgD}$ $(+)$ increased significantly upon vaccination in the second measurement only in patients with mild cGVHD $(\mathrm{p}<0.001)$, in contrast to patients with a moderate or severe form. We observed slightly lower median values of DN IgD-CD27- in patients without cGVHD and with a mild form of cGVHD $4.74 \%$ and $4.51 \%$, respectively, while in patients with a history of moderate and severe cGVHD had median values $6.27 \%$ and $5.47 \%$ without statistical significance.

The rate of CD8(-) cells with IFN $\gamma$ expression had no statistical impact on vaccination efficacy and did not change during vaccination. In contrast, the mean percentage of CD8(-) IL2(+) cells differed significantly between the WR group and VGR group (18.9 vs. 26.9\%; $\mathrm{p}=0.043$ ) and decreased upon immunization in all patients. The mean values of CD8(-)IL2 (+) cells decreased upon vaccination in patients without cGVHD and with a mild form of cGVHD (23.8 and $20.1 \%$ vs. 16.1 and $14.7 \%$, respectively), while these values remained at a stable level of approximately $20 \%$ in patients with a history of severe cGVHD. The rates of IL4-expressing cells increased gradually in the WR group upon vaccination in subsequent analyses $(2.3$ vs. 2.9 vs. $3.2 \% ; \mathrm{p}=0.033)$. In patients with severe $c G V H D$, a similar increase in the IL4(+) cell rate was observed during vaccination ( 2.6 vs. 3.3 vs. $3.5 \%$; $=0.012, p=0.024$ ). The rate of IL5(+) T lymphocytes rose already in the second measurement in GRs $(\mathrm{p}=0.023)$ and VGRs $(\mathrm{p}<0.001)$, and a delayed rise after vaccination completion was observed in WRs $(p=0.01)$. In patients with mild cGVHD or without a history of cGVHD, the IL5 $(+)$ cell rate increased in the second measurement $(\mathrm{p}=$ 0.027 and $\mathrm{p}<0.001$, respectively), whereas a delayed rise was present in patients with moderate and severe cGVHD $(\mathrm{p}=0.017$ and $\mathrm{p}=0.038$, respectively).

\section{DISCUSSION}

Despite the high immunogenicity of the anti-HBV vaccine, approximately $5 \%$ of healthy vaccinees fail to mount an adequate humoral response. The response rate in immunocompromised patients is reported to be lower. Preceding therapy, a vaccination schedule, and a dose of antigen are postulated to have a significant impact on immune responses (34). The immunogenicity of the 
TABLE 4 | Detailed comparison of general characteristics and parameters describing immune reconstitution between the early vaccination group and the late vaccination group.

\begin{tabular}{|c|c|c|c|c|}
\hline & $\begin{array}{c}\text { Early group }<24 \\
\text { months post-HCT }\end{array}$ & $\begin{array}{c}\text { Late group>24 } \\
\text { months post-HCT }\end{array}$ & Units & Statistics \\
\hline \multicolumn{5}{|l|}{ General characteristics } \\
\hline Female/male & $42 / 58$ & $47 / 53$ & $\%$ & $p=N S$ \\
\hline Age at vaccination* & $35(21-50)$ & $43(20-57)$ & years & $p=0.002$ \\
\hline Time after $\mathrm{HCT}^{\star}$ & $20(12-24)$ & $56(31-119)$ & months & $\mathrm{N} / \mathrm{A}$ \\
\hline Time after immunosuppression taper ${ }^{\star}$ & $7(2-19)$ & $20(12-31)$ & months & N/A \\
\hline \multicolumn{5}{|l|}{ Immunoglobulins } \\
\hline $\lg G^{\star \star}$ & $11.36( \pm 4.11)$ & $11.55( \pm 2.56)$ & $g / L$ & $p=N S$ \\
\hline $\lg A^{\star \star}$ & $1.36( \pm 0.68)$ & $2.1( \pm 0.97)$ & $g / L$ & $p=0.005$ \\
\hline $\lg M^{\star \star}$ & $0.81( \pm 0.42)$ & $0.96( \pm 0.45)$ & $g / L$ & $p=N S$ \\
\hline \multicolumn{5}{|l|}{ Lymphocytes } \\
\hline Absolute lymphocyte count*^ & $2.05( \pm 0.91)$ & $2.42( \pm 0.91)$ & $\times 10^{9} / \mathrm{L}$ & $p=N S$ \\
\hline B lymphocytes CD19+* & 0.28 & 0.41 & $\times 10^{9} / \mathrm{L}$ & $p=N S$ \\
\hline $\mathrm{CD} 19+\lg \mathrm{D}(+)^{*}$ & 0.11 & 0.16 & $\times 10^{9} / \mathrm{L}$ & $p=N S$ \\
\hline CD19+lgM $(+)^{*}$ & 0.21 & 0.31 & $\times 10^{9} / \mathrm{L}$ & $\mathrm{p}=\mathrm{NS}$ \\
\hline $\mathrm{CD} 19+\lg G(+)^{*}$ & 0.2 & 0.1 & $\%$ & $p=N S$ \\
\hline \multirow{2}{*}{ Naïve CD19+lgM(+) CD27-* } & 50.0 & 42.6 & $\%$ & $p=N S$ \\
\hline & 0.14 & 0.17 & $\times 10^{9} / \mathrm{L}$ & $\mathrm{p}=\mathrm{NS}$ \\
\hline \multirow[t]{2}{*}{ Naïve CD19+lgD(+) CD27-* } & 81.0 & 83.15 & $\%$ & $\mathrm{p}=\mathrm{NS}$ \\
\hline & 0.23 & 0.34 & $\times 10^{9} / \mathrm{L}$ & $p=N S$ \\
\hline Memory CD19+lgG(+) CD27+* & 0.6 & 0.25 & $\%$ & $p=N S$ \\
\hline \multirow[t]{2}{*}{ Memory CD19+lgM(+) CD27+* } & 2.5 & 2.7 & $\%$ & $p=N S$ \\
\hline & 0.007 & 0.11 & $\times 10^{9} / \mathrm{L}$ & $\mathrm{p}=\mathrm{NS}$ \\
\hline \multirow[t]{2}{*}{ Memory CD19+lgD(+) CD27+* } & 2.0 & 2.7 & $\%$ & $p=N S$ \\
\hline & 0.006 & 0.11 & $\times 10^{9} / \mathrm{L}$ & $p=N S$ \\
\hline \multirow[t]{2}{*}{ Memory CD19+lgD(-) CD27(-)* } & 5.4 & 5.12 & $\%$ & $p=N S$ \\
\hline & 0.015 & 0.014 & $\times 10^{9} / \mathrm{L}$ & $\mathrm{p}=\mathrm{NS}$ \\
\hline T lymphocytes CD3+* & 1.07 & 1.25 & $\times 10^{9} / \mathrm{L}$ & $p=N S$ \\
\hline T lymphocytes CD3+CD4+* & 0.33 & 0.53 & $\times 10^{9} / \mathrm{L}$ & $p=0.006$ \\
\hline T lymphocytes CD3+CD8+* & 0.73 & 0.7 & $\times 10^{9} / \mathrm{L}$ & $p=N S$ \\
\hline CD4/CD8 ratio* & 0.52 & 0.86 & & $p=0.008$ \\
\hline \multirow[t]{2}{*}{ Naïve CD4+CD45RA + ${ }^{*}$} & 25.0 & 20.4 & $\%$ & $p=N S$ \\
\hline & 0.08 & 0.1 & $\times 10^{9} / \mathrm{L}$ & $p=0.096$ \\
\hline \multirow[t]{2}{*}{ Memory CD4+CD45RO+* } & 61.0 & 65.9 & $\%$ & $p=N S$ \\
\hline & 0.21 & 0.34 & $\times 10^{9} / \mathrm{L}$ & $p=0.011$ \\
\hline \multirow[t]{2}{*}{ Naïve CD8+CD45RA+* } & 41.1 & 44.9 & $\%$ & $p=N S$ \\
\hline & 0.29 & 0.3 & $\times 10^{9} / \mathrm{L}$ & $p=N S$ \\
\hline \multirow[t]{2}{*}{ Memory CD8+CD45RO+* } & 26.3 & 26.5 & $\%$ & $p=N S$ \\
\hline & 0.2 & 0.19 & $\times 10^{9} / \mathrm{L}$ & $p=N S$ \\
\hline \multicolumn{5}{|l|}{ Th1 cytokine expression* } \\
\hline $\mathrm{CD} 3+\mathrm{CD} 8-\mathrm{INF} \gamma+$ & 20.34 & 20.22 & $\%$ & $p=N S$ \\
\hline CD3+CD8-IL2+ & 24.13 & 26.17 & $\%$ & $p=N S$ \\
\hline $\mathrm{CD} 3+\mathrm{CD} 8+\mathrm{INF} \gamma+$ & 34.23 & 27.83 & $\%$ & $\mathrm{p}=0.052$ \\
\hline CD3+CD8+IL2+ & 6.16 & 5.0 & $\%$ & $p=N S$ \\
\hline $\mathrm{CD} 3+$ IL2- INF $\gamma+$ & 49.14 & 38.13 & $\%$ & $p=0.018$ \\
\hline $\mathrm{CD} 3+\mathrm{IL} 2+\mathrm{INF} \gamma-$ & 14.74 & 21.62 & $\%$ & $\mathrm{p}=0.011$ \\
\hline \multicolumn{5}{|l|}{ Th2 cytokine expression* } \\
\hline CD3+CD8-IL4+ & 1.79 & 2.15 & $\%$ & $p=N S$ \\
\hline CD3+CD8-IL5+ & 0.09 & 0.15 & $\%$ & $p=N S$ \\
\hline CD3+CD8-IL10+ & 0.12 & 0.12 & $\%$ & $p=N S$ \\
\hline CD3+CD8+IL4+ & 0.41 & 0.48 & $\%$ & $p=N S$ \\
\hline CD3+CD8+IL5+ & 0.05 & 0.04 & $\%$ & $p=N S$ \\
\hline CD3+CD8+IL10+ & 0.04 & 0.03 & $\%$ & $p=N S$ \\
\hline
\end{tabular}

*Median value (range in parenthesis).

${ }^{* *}$ Arithmetic mean value.

Bolded values showed statistical differences.

primary vaccination is known to last 10 to 31 years, but not in the case of immunocompromised patients (34). In the analysis performed by Kaloyannidis et al., the probability of losing HBV immunity was $100 \%$ at 5 years post-HCT for patients who received transplants from nonimmunized donors and $78 \%$ and $58 \%$ for those who received transplants from vaccinated donors and naturally immunized donors, respectively (6). In contrast to our study group, those patients were not revaccinated post-HCT, and those data cannot be directly compared to our results. The aforementioned study by Kaloyannidis et al. also reported a high 
probability of reversed seroconversion, reaching $18 \%$ at 12 years (6). In the analysis by Mikulska et al., HBV reactivations were observed in $10 \%$ of patients with a median time of 19 months post-HCT (8). In our study group, from 5 patients with naturally acquired immunity, no reverse seroconversion was observed. Two patients received booster doses of rHBsAg as prevention when a gradual drop in anti-HBs Abs was observed.

A detailed analysis of our data from the early posttransplant period showed an evident impact of either patients' or their donors' vaccination status before HCT. In the case of insufficient or no protection against $\mathrm{HBV}$, passive immunization remains the only solution. However, passively transferred anti-HBs Abs have a limited lifespan, as shown in our study. Therefore, repetitive infusions would be required to preserve sufficient protection. More prolonged protective anti-HBs titers were observed in patients effectively vaccinated before HCT and in those who received transplants from vaccinated donors. Moreover, donor immunization provides an additional benefit for an HCT recipient - memory $\mathrm{B}$ and $\mathrm{T}$-cells responsible for the adoptive transfer of immunity, which can be easily recalled by a booster dose of vaccine $(34,35)$.

The ORR to post-HCT vaccination of $100 \%$ and the longlasting maintenance of anti-HBV immunity might be astonishing given that these vaccinations were administered to immunocompromised patients. Similar results were reported by Machado et al. in 45 recipients immunized $\geq 1$ year after alloHCT, while in another cited study, the seroconversion rate in 168 adult patients was $59 \%(13,36)$. We used three known strategies to improve the immune response: an increased vaccine dose to $40 \mu \mathrm{g}$ administered until seroconversion was achieved, an intensified dosing schedule and the co-administration of rHBsAg with other vaccines from the vaccination protocol (34). A higher dose of the vaccine administered initially augments the B-cell response and increases the proportion of memory B-cells, which could also have an impact on the duration of long-lasting immunity (37). Moreover, in the study group, more than $90 \%$ of the VGRs received transplants from vaccinated donors, thereby transferring memory cells to their recipients. The positive effect of prior donor immunization was also confirmed in the multivariate analysis.

The median follow-up in our study, exceeding 10 years, enabled the monitoring of a long-lasting anti-HBV immune status in the majority of patients. The inevitable loss of anti-HBV immunity was confirmed in HCT recipients, but the rate was dependent on the degree of the immune response to the primary inoculum series (37). No patient from the VGR group required any booster dose of rHBsAg, and only $20 \%$ of patients with previously noted adoptive immunity transfer required a booster dose. In contrast, primary WRs, according to our predefined criteria, should be closely monitored as booster doses of rHBsAg are likely to be needed. However, one can expect an intense reaction of memory cells to recall antigen, as in most cases, one booster dose was sufficient to achieve anti-HBs Abs titers $>100 \mathrm{mIU} / \mathrm{ml}$.

Upon injection, rHBsAg is lysed and processed by antigenpresenting specific B-cells and presented with MHC-II molecule to Th2 cells. Activated Th2 lymphocytes induce differentiation of
B-cells to plasma cells, secreting HBsAg in high quantities to stimulate immune B-cell and T-cell memory (33). To better understand the immune response in HCT recipients, we measured several parameters of the immune system, including subpopulations of $\mathrm{B}$ and T-cells, with deep analysis of subsets expressing Th1 and Th2 cytokine profiles. We confirmed that it is optimal to start active immunization when the absolute CD4(+) T cell count exceeds $0.2 \times 10^{9} / \mathrm{L}$. Similar to HCT recipients, in patients with HIV infection, the seroconversion rate after $\mathrm{HBV}$ vaccination was shown to be directly proportional to the CD4(+) cell count (34). The repertoire of naïve $\mathrm{T}$ lymphocytes is crucial for the optimal response to antigens and depends on thymus regeneration starting from 6 to 12 months posttransplantation (38). In our study group, there was a trend toward a weaker response in those with naïve CD4 (+) cells below $0.03 \times 10^{9} / \mathrm{L}$, which was already reported by Roux et al. in HCT recipients vaccinated with tetanus toxoid (14). We did not observe a decreased proportion of Th2-like cytokine-producing CD4 + cells, while IL2-producing CD4+ cells were significantly lower in WRs, as described in "in vitro" studies (39). Moreover, the realization of an active immunization protocol led to a gradual increase in IL4(+) and IL5(+) cells and a decrease in IL2(+)CD4+ lymphocytes.

The early vaccination group had significantly lower IgA immunoglobulin levels than the late group, which could reflect an impaired isotype switch from naïve to memory B-cells. However, we did not confirm the influence of median IgA levels on the quality of the postvaccination humoral response $(15,16)$. Differences in circulating B-cells at the time of vaccination did not show significant impact on the immune response (15). However, the humoral response was parallel with the increase in memory $\operatorname{IgM}(+)$ and $\operatorname{IgD}(+) \mathrm{B}$-cell subsets. Interestingly, we found a significantly higher percentage of late differentiated memory DN B lymphocytes in WRs. This subpopulation is reported to reflect senescence of the immune system related to chronic inflammatory processes, e.g., HIV infection, lupus, Alzheimer's disease (40). This observation could be explained by a high rate of patients with a history of severe cGVHD in the WR group.

The comparison of the early and late groups showed some differences in immune reconstitution but these parameters had ultimately no impact on the quality of the post-vaccination response in our study group neither in the univariate nor multivariate analysis. In multivariate analysis, there was only a slight trend toward less common weak responses to immunization with rHBsAg in the late vaccination group. This is another suggestion that vaccination against hepatitis B started at 6 months post-HCT according to the international recommendations is absolutely rational.

Severe GVHD induces structural damage that has a serious and durable impact on thymus functioning and output (41-43). A direct influence of immunosuppressive agents in our study group was excluded since immunosuppression was discontinued at least 2 months before enrollment. However, a history of severe cGVHD significantly influenced the response to anti-HBV vaccination and the maintenance of protective immunity, even though immunization was performed in the nonactive phase of the disease. We also noted a delayed increase in Th2 IL4(+) and IL5(+) cells in response to rHBsAg in WRs and patients with a 
history of severe cGVHD. Our observations are in line with those of the study by Jaffe et al., in which the seroconversion after 3 doses of rHBsAg was observed in $64 \%$ of patients after HCT, and the negative influence of a GVHD history was also demonstrated (13). Furthermore, Kaloyannidis et al. indicated cGVHD as an independent factor for anti-HBs Ab disappearance (6).

Guidelines for the prevention of infectious complications among HCT recipients recommend the same vaccination schedule for all HCT recipients, and active immunization of patients with cGVHD should not be postponed $(18,20,21,36,38)$. Evidence exists that severe $\mathrm{cGVHD}$ and its treatment deteriorate the efficacy of active immunization $(14,18)$. Therefore, immunization should not begin during the exacerbation of GvHD and the escalation of immunosuppressive treatment. Instead, effective anti-infectious prophylaxis should be provided (18).

Our study has several limitations. First, a laboratory part was designed and performed when the serological assessment of antibody response was only available for vaccination against $\mathrm{HBV}$ in the hospital laboratory. Therefore, we did not analyze the response rates to the remaining co-administered vaccines. Second, although we did not observe any case of hepatitis B in our study group, which gives evidence for clinical protection of patients with serological response to vaccination, we did not investigate the specific cellular immunity. The modern assays such as Ag-specific cell detection, protein quantification, and transcriptomics techniques would give a deeper insight into postvaccine immunity and potential correlation between the humoral and cellular anti-viral protection. Third, immunophenotyping was performed with the use of 3-color flow cytometry as at the time of laboratory analyses it was the only available equipment. The multicolor flow cytometry available nowadays would extend the analytic possibilities.

In summary, vaccination of recipients and their donors against $\mathrm{HBV}$ prior to HCT is beneficial in many aspects, including protection in the early posttransplantation period. Our results add important information that might help the clinical management of HCT recipients by implementing a double dose of rHBsAg $(40 \mu \mathrm{g})$ in patients with a history of cGVHD and those not immunized before HCT or those who received transplants from nonimmunized donors until seroconversion is achieved. The subsequent doses might be reduced to the standard $20 \mu \mathrm{g}$. Second, an intensified vaccination schedule of 0-1-2-6-(12) months is advised for WRs who do not achieve a protective anti$\mathrm{HBs} \mathrm{Ab}$ titer $>10 \mathrm{mIU} / \mathrm{ml}$ or in those for whom the level is between 10 and $100 \mathrm{mIU} / \mathrm{ml}$ after 3 doses of HBV vaccine. Third, a titer of anti-HBs Abs should be monitored routinely in WRs and patients with cGVHD, since a decline in specific Abs requiring reimmunization is expected. Finally, in GRs and VGRs and patients without cGVHD, the titer of anti-HBs Abs can be measured in longer (e.g., 5-year) intervals.

\section{REFERENCES}

1. Passweg JR, Baldomero H, Bader P, Bonini C, Cesaro S, Dreger P, et al. Hematopoietic stem cell transplantation in Europe 2014: more than 40000

\section{DATA AVAILABILITY STATEMENT}

The original contributions presented in the study are included in the article/supplementary materials. Further inquiries can be directed to the corresponding author/s.

\section{ETHICS STATEMENT}

The studies involving human participants were reviewed and approved by Independent Bioethics Committee of the Medical University of Gdansk. The patients/participants provided their written informed consent to participate in this study.

\section{AUTHOR CONTRIBUTIONS}

AP was involved in the conception and design of the study, the acquisition of data, statistical analysis, and the analysis and interpretation of data, and writing of the manuscript. PW took part in the statistical analysis and critical revision. LG was involved in the data analysis and critical revision. KL took part in the data analysis, drafting of the article, and critical revision. PT was involved in the data analysis and critical revision. $\mathrm{MB}$ took part in the data analysis and critical revision. JZ was involved in the data analysis, drafting of the article, and critical review. All authors contributed to the article and approved the submitted version.

\section{FUNDING}

The laboratory analyses (immunophenotype of lymphocyte subsets and cytokine expression) were financed by a grant from the Ministry of Science and Higher Education (Mentorship Grant number 2 PO5B 080 26). This work was supported by the French Government and the French Embassy in Poland (science.varsovieamba@diplomatie.gouv.fr). The competence to assess Th1 and Th2 cytokine expression was gained thanks to the French Government Scholarship (Number 385816B/P361471J) taking place in Laboratoire d'Immunology CHU Rangueil, Toulouse, France, in 2003.

\section{ACKNOWLEDGMENTS}

To the former teams of the Laboratoire d'Immunology CHU Rangueil, supervised by Dr. Jean Tkaczuk, and the Laboratorium Cytomorfologii i Cytometrii, UCK Gdansk, supervised by Dr hab. Krzysztof Lewandowski.

transplants annually. Bone Marrow Transplant (2016) 51(6):786-92. doi: 10.1038/bmt.2016.20

2. Gratwohl A, Brand R, Frassoni F, Rocha V, Niederwieser D, Reusser P, et al. Cause of death after allogeneic haematopoietic stem cell transplantation (HSCT) in early leukaemias: an EBMT analysis of lethal infectious 
complications and changes over calendar time. Bone Marrow Transplant (2005) 36(9):757-69. doi: 10.1038/sj.bmt.1705140

3. Seggewiss R, Einsele H. Immune reconstitution after allogeneic transplantation and expanding options for immunomodulation: an update. Blood (2010) 115(19):3861-8. doi: 10.1182/blood-2009-12-234096

4. Storek J, Dawson MA, Storer B, Stevens-Ayers T, Maloney DG, Marr KA, et al. Immune reconstitution after allogeneic marrow transplantation compared with blood stem cell transplantation. Blood (2001) 97(11):33809. doi: 10.1182/blood.V97.11.3380

5. Storek J, Joseph A, Espino G, Dawson MA, Douek DC, Sullivan KM, et al. Immunity of patients surviving 20 to 30 years after allogeneic or syngeneic bone marrow transplantation. Blood (2001) 98(13):3505-12. doi: 10.1182/ blood.V98.13.3505

6. Kaloyannidis P, Batsis I, Yannaki E, Adamidou D, Bartzoudis D, Papathanasiou M, et al. Allografted recipients immunized against hepatitis $B$ virus are at high risk of gradual surface antibody (HbsAb) disappearance post transplant, regardless of adoptive immunity transfer. Biol Blood Marrow Transplant (2007) 13(9):1049-56. doi: 10.1016/j.bbmt.2007.05.009

7. Parkkali T, Ruutu T, Stenvik M, Kuronen T, Kayhty H, Hovi T, et al. Loss of protective immunity to polio, diphtheria and Haemophilus influenzae type $\mathrm{b}$ after allogeneic bone marrow transplantation. APMIS (1996) 104(5):383-8. doi: 10.1111/j.1699-0463.1996.tb00731.x

8. Mikulska M, Nicolini L, Signori A, Rivoli G, Del Bono V, Raiola AM, et al. Hepatitis B reactivation in HBsAg-negative/HBcAb-positive allogeneic haematopoietic stem cell transplant recipients: risk factors and outcome. Clin Microbiol Infect (2014) 20(10):O694-70. doi: 10.1111/1469-0691.12611

9. Vigano M, Vener C, Lampertico P, Annaloro C, Pichoud C, Zoulim F, et al. Risk of hepatitis B surface antigen seroreversion after allogeneic hematopoietic SCT. Bone Marrow Transplant (2011) 46(1):125-31. doi: $10.1038 / \mathrm{bmt} .2010 .70$

10. Hamaguchi M, Yamada H, Gondo H, Takemoto Y, Morishima Y, Kodera Y. Retrospective study on the impact of hepatitis $\mathrm{B}$ and hepatitis $\mathrm{C}$ virus infection on hematopoietic stem cell transplantation in Japan. Int J Hematol (2002) 75 (3):324-31. doi: 10.1007/BF02982051

11. Avigan D, Pirofski LA, Lazarus HM. Vaccination against infectious disease following hematopoietic stem cell transplantation. Biol Blood Marrow Transplant (2001) 7(3):171-83. doi: 10.1053/bbmt.2001.v7.pm11302551

12. Conrad A, Alcazer V, Valour F, Ader F, Lyon HSG. Vaccination postallogeneic hematopoietic stem cell transplantation: what is feasible? Expert Rev Vaccines (2018) 17(4):299-309. doi: 10.1080/14760584.2018.1449649

13. Jaffe D, Papadopoulos EB, Young JW, O’Reilly RJ, Prockop S, Kernan NA, et al. Immunogenicity of recombinant hepatitis $\mathrm{B}$ vaccine (rHBV) in recipients of unrelated or related allogeneic hematopoietic cell (HC) transplants. Blood (2006) 108(7):2470-5. doi: 10.1182/blood-2006-04-006981

14. Roux E, Dumont-Girard F, Starobinski M, Siegrist CA, Helg C, Chapuis B, et al. Recovery of immune reactivity after T-cell-depleted bone marrow transplantation depends on thymic activity. Blood (2000) 96(6):2299-303. doi: 10.1182/blood.V96.6.2299.h8002299_2299_2303

15. D’Orsogna LJ, Wright MP, Krueger RG, McKinnon EJ, Buffery SI, Witt CS, et al. Allogeneic hematopoietic stem cell transplantation recipients have defects of both switched and igm memory B cells. Biol Blood Marrow Transplant (2009) 15(7):795-803. doi: 10.1016/j.bbmt.2008.11.024

16. Carpenter PA, Englund JA. How I vaccinate blood and marrow transplant recipients. Blood (2016) 127(23):2824-32. doi: 10.1182/blood-2015-12550475

17. Cordonnier C, Einarsdottir S, Cesaro S, Di Blasi R, Mikulska M, Rieger C, et al. Vaccination of haemopoietic stem cell transplant recipients: guidelines of the 2017 European Conference on Infections in Leukaemia (ECIL 7). Lancet Infect Dis (2019) 19(6): E200-12. doi: 10.1016/S1473-3099(18)30600-5

18. Hilgendorf I, Freund M, Jilg W, Einsele H, Gea-Banacloche J, Greinix H, et al. Vaccination of allogeneic haematopoietic stem cell transplant recipients: report from the international consensus conference on clinical practice in chronic GVHD. Vaccine (2011) 29(16):2825-33. doi: 10.1016/j.vaccine. 2011.02.018

19. Ljungman P. Vaccination of immunocompromised patients. Clin Microbiol Infect (2012) 18(Suppl 5):93-9. doi: 10.1111/j.1469-0691.2012.03971.x

20. Rubin LG, Levin MJ, Ljungman P, Davies EG, Avery R, Tomblyn M, et al. 2013 IDSA clinical practice guideline for vaccination of the immunocompromised host. Clin Infect Dis (2014) 58(3):309-18. doi: $10.1093 / \mathrm{cid} / \mathrm{cit} 816$

21. Ullmann AJ, Schmidt-Hieber M, Bertz H, Heinz WJ, Kiehl M, Kruger W, et al. Infectious diseases in allogeneic haematopoietic stem cell transplantation: prevention and prophylaxis strategy guidelines 2016. Ann Hematol (2016) 95 (9):1435-55. doi: 10.1007/s00277-016-2711-1

22. Krenger W, Hollander GA. The immunopathology of thymic GVHD. Semin Immunopathol (2008) 30(4):439-56. doi: 10.1007/s00281-008-0131-6

23. Zeiser R, Blazar BR. Pathophysiology of Chronic Graft-versus-Host Disease and Therapeutic Targets. N Engl J Med (2017) 377(26):2565-79. doi: 10.1056/ NEJMra1703472

24. Perez-Simon JA, Sanchez-Abarca I, Diez-Campelo M, Caballero D, San Miguel J. Chronic graft-versus-host disease: Pathogenesis and clinical management. Drugs (2006) 66(8):1041-57. doi: 10.2165/00003495200666080-00002

25. Svegliati S, Olivieri A, Campelli N, Luchetti M, Poloni A, Trappolini S, et al. Stimulatory autoantibodies to PDGF receptor in patients with extensive chronic graft-versus-host disease. Blood (2007) 110(1):237-41. doi: 10.1182/ blood-2007-01-071043

26. Jagasia MH, Greinix HT, Arora M, Williams KM, Wolff D, Cowen EW, et al. National Institutes of Health Consensus Development Project on Criteria for Clinical Trials in Chronic Graft-versus-Host Disease: I. The 2014 Diagnosis and Staging Working Group report. Biol Blood Marrow Transplant (2015) 21 (3):389-401.e1. doi: 10.1016/j.bbmt.2014.12.001

27. Cuthbert RJ, Iqbal A, Gates A, Toghill PJ, Russell NH. Functional hyposplenism following allogeneic bone marrow transplantation. J Clin Pathol (1995) 48(3):257-9. doi: 10.1136/jcp.48.3.257

28. Kulkarni S, Powles R, Treleaven J, Riley U, Singhal S, Horton C, et al. Chronic graft versus host disease is associated with long-term risk for pneumococcal infections in recipients of bone marrow transplants. Blood (2000) 95 (12):3683-6. doi: 10.1182/blood.V95.12.3683.012k19_3683_3686

29. Witherspoon RP, Storb R, Ochs HD, Fluornoy N, Kopecky KJ, Sullivan KM, et al. Recovery of antibody production in human allogeneic marrow graft recipients: influence of time posttransplantation, the presence or absence of chronic graft-versus-host disease, and antithymocyte globulin treatment. Blood (1981) 58(2):360-8. doi: 10.1182/blood.V58.2.360.bloodjournal582360

30. Jung T, Schauer U, Heusser C, Neumann C, Rieger C. Detection of intracellular cytokines by flow cytometry. J Immunol Methods (1993) 159 (1-2):197-207. doi: 10.1016/0022-1759(93)90158-4

31. Rostaing L, Tkaczuk J, Durand M, Peres C, Durand D, de Preval C, et al. Kinetics of intracytoplasmic Th1 and Th2 cytokine production assessed by flow cytometry following in vitro activation of peripheral blood mononuclear cells. Cytometry (1999) 35(4):318-28. doi: 10.1002/(SICI) 1097-0320 (19990401)35:4<318::AID-CYTO4>3.0.CO;2-4

32. Bosch M, Khan FM, Storek J. Immune reconstitution after hematopoietic cell transplantation. Curr Opin Hematol (2012) 19(4):324-35. doi: 10.1097/ MOH.0b013e328353bc7d

33. Bauer T, Jilg W. Hepatitis B surface antigen-specific T and B cell memory in individuals who had lost protective antibodies after hepatitis $\mathrm{B}$ vaccination. Vaccine (2006) 24(5):572-7. doi: 10.1016/j.vaccine.2005.08.058

34. Walayat S, Ahmed Z, Martin D, Puli S, Cashman M, Dhillon S. Recent advances in vaccination of non-responders to standard dose hepatitis $B$ virus vaccine. World J Hepatol (2015) 7(24):2503-9. doi: 10.4254/ wjh.v7.i24.2503

35. Harris AE, Styczynski J, Bodge M, Mohty M, Savani BN, Ljungman P. Pretransplant vaccinations in allogeneic stem cell transplantation donors and recipients: an often-missed opportunity for immunoprotection? Bone Marrow Transplant (2015) 50(7):899-903. doi: 10.1038/bmt.2015.49

36. Small TN, Cowan MJ. Immunization of hematopoietic stem cell transplant recipients against vaccine-preventable diseases. Expert Rev Clin Immunol (2011) 7(2):193-203. doi: 10.1586/eci.10.103

37. Koff RS. Immunogenicity of hepatitis $B$ vaccines: implications of immune memory. Vaccine (2002) 20(31-32):3695-701. doi: 10.1016/S0264-410X(02) 00405-X

38. Tomblyn M, Chiller T, Einsele H, Gress R, Sepkowitz K, Storek J, et al. Guidelines for preventing infectious complications among hematopoietic cell transplantation recipients: a global perspective. Biol Blood Marrow Transplant (2009) 15(10):1143-238. doi: 10.1016/j.bbmt.2009.06.019 
39. Kardar GA, Jeddi-Tehrani M, Shokri F. Diminished Th1 and Th2 cytokine production in healthy adult nonresponders to recombinant hepatitis B vaccine. Scand J Immunol (2002) 55(3):311-4. doi: 10.1046/j.13653083.2002.01057.x

40. Martorana A, Balistreri CR, Bulati M, Buffa S, Azzarello DM, Camarda C, et al. Double negative (CD19+IgG+IgD-CD27-) B lymphocytes: a new insight from telomerase in healthy elderly, in centenarian offspring and in Alzheimer's disease patients. Immunol Lett (2014) 162(1 Pt B):303-9. doi: 10.1016/ j.imlet.2014.06.003

41. Weinberg K, Blazar BR, Wagner JE, Agura E, Hill BJ, Smogorzewska M, et al. Factors affecting thymic function after allogeneic hematopoietic stem cell transplantation. Blood (2001) 97(5):1458-66. doi: 10.1182/blood.V97.5.1458

42. Hauri-Hohl MM, Keller MP, Gill J, Hafen K, Pachlatko E, Boulay T, et al. Donor T-cell alloreactivity against host thymic epithelium limits T-cell development after bone marrow transplantation. Blood (2007) 109(9):4080-8. doi: 10.1182/blood-2006-07-034157
43. Clave E, Busson M, Douay C, Peffault de Latour R, Berrou J, Rabian C, et al. Acute graft-versus-host disease transiently impairs thymic output in young patients after allogeneic hematopoietic stem cell transplantation. Blood (2009) 113(25):6477-84. doi: 10.1182/blood-2008-09-176594

Conflict of Interest: The authors declare that the research was conducted in the absence of any commercial or financial relationships that could be construed as a potential conflict of interest.

Copyright (C) 2020 Piekarska, Wisniewski, Lewandowski, Gil, Trzonkowski, Bieniaszewska and Zaucha. This is an open-access article distributed under the terms of the Creative Commons Attribution License (CC BY). The use, distribution or reproduction in other forums is permitted, provided the original author(s) and the copyright owner(s) are credited and that the original publication in this journal is cited, in accordance with accepted academic practice. No use, distribution or reproduction is permitted which does not comply with these terms. 